\title{
Implementing the Fatigue Guidelines at One NCCN Member Institution: Process and Outcomes
}

Tami Borneman, MSN, RN, CNS; ${ }^{a}$ Barbara F. Piper, DNSc, RN, AOCN; ${ }^{c}$ Virginia Chih-Yi Sun, MSN, RN, ANP; Marianna Koczywas, MD; ${ }^{\mathrm{b}}$ Gwen Uman, PhD, RN; ${ }^{\mathrm{a}}$ and Betty Ferrell, PhD, RN; ${ }^{a}$ Duarte and Los Angeles, California, and Scottsdale, Arizona

\section{Key Words}

Fatigue, patient barriers, provider barriers, system barriers, evidencebased guidelines

\begin{abstract}
Fatigue, despite being the most common and distressing symptom in cancer, is often unrelieved because of numerous patient, provider, and system barriers. The overall purpose of this 5-year prospective clinical trial is to translate the NCCN Cancer-Related Fatigue Clinical Practice Guidelines in Oncology and NCCN Adult Cancer Pain Clinical Practice Guidelines in Oncology into practice and develop a translational interventional model that can be replicated across settings. This article focuses on one NCCN member institution's experience related to the first phase of the NCCN Cancer-Related Fatigue Guidelines implementation, describing usual care compared with evidence-based guidelines. Phase 1 of this 3-phased clinical trial compared the usual care of fatigue with that administered according to the NCCN guidelines. Eligibility criteria included age 18 years or older; English-speaking; diagnosed with breast, lung, colon, or prostate cancer; and fatigue and/or pain ratings of 4 or more on a 0 to 10 screening scale. Research nurses screened all available subjects in a cancer center medical oncology clinic to identify those meeting these criteria. Instruments included the Piper Fatigue Scale, a Fatigue Barriers Scale, a Fatigue Knowledge Scale, and a Fatigue Chart Audit Tool. Descriptive and inferential statistics were used in
\end{abstract}

From the apepartment of Nursing Research \& Education, Division of Population Sciences, Beckman Research Institute, and 'Department of Medical Oncology and Therapeutics Research, City of Hope National Medical Center, Duarte, California; 'Scottsdale Healthcare, University of Arizona, Scottsdale, Arizona; and 'Vital Research, LLC, Los Angeles, California.

The authors have no financial interest, arrangement, or affiliation with the manufacturers of any products discussed in the article or their competitors. Submitted April 11, 2007; accepted for publication August 13, 2007.

Correspondence: Tami Borneman, MSN, RN, CNS, Department of Nursing Research \& Education, Division of Population Sciences, Beckman Research Institute, City of Hope National Medical Center, 1500 E. Duarte Road, Duarte, CA 91010. E-mail: tborneman@coh.org data analysis. At baseline, 45 patients had fatigue only $(\geq 4)$ and 24 had both fatigue and pain $(\geq 4)$. This combined sample $(N=69)$ was predominantly Caucasian (65\%), female (63\%), an average of 60 years old, diagnosed with stage 3 or 4 breast cancer, and undergoing treatment $(82 \%)$. The most common barriers noted were patients' belief that physicians would introduce the subject of fatigue if it was important (patient barrier); lack of fatigue documentation (professional barrier); and lack of supportive care referrals (system barrier). Findings showed several patient, professional, and system barriers that distinguish usual care from that recommended by the NCCN Cancer-Related Fatigue Guidelines. Phase 2, the intervention model, is designed to decrease these barriers and improve patient outcomes over time, and is in progress. (JNCCN 2007;5:1092-1101)

\section{Background}

Fatigue, the most common and distressing symptom in cancer, ${ }^{1}$ remains poorly managed despite the existence of the NCCN Cancer-Related Fatigue Clinical Practice Guidelines in Oncology. These deficiencies in fatigue management are caused by numerous patient, provider, and system barriers. ${ }^{2}$

\section{Purpose}

The overall purpose of this 5-year prospective clinical trial funded by the National Cancer Institute (NCI) is to 1) translate the NCCN Cancer-Related Fatigue Clinical Practice Guidelines in Oncology and NCCN Adult Cancer Pain Clinical Practice Guidelines in Oncology into practice and 2) develop a translational intervention model based on the guidelines that can be replicated across settings through reducing patient, professional, and system barriers to improve outcomes. The entire trial is being implemented over 3 phases. Phase 1 documents usual care at baseline and 1 and 3 months later. Phase 2, 
Implementing the NCCN Fatigue Guidelines

the "high-intensity" translational intervention model, involves systematic patient and physician educational sessions augmented by peer audit and feedback on changes in patients' fatigue and pain status. This phase also examines guideline adherence measured at baseline and 1 and 3 months later. Phase 3, the "low-intensity" intervention, involves translation of the model into practice by hospital staff. This article describes one NCCN member institution's baseline findings related to the first phase of the NCCN Cancer-Related Fatigue Guidelines implementation, describing usual care compared with evidence-based guidelines.

\section{Review of the Literature}

The symptom of fatigue has emerged as a high-priority concern in cancer. ${ }^{3-6}$ In 2002, an NIH Consensus and State-of-the-Science conference was held on symptom management in cancer that addressed pain, depression, and fatigue. ${ }^{2}$ Recommendations for treating fatigue included 1) using a brief assessment tool to routinely ask patients about fatigue and initiate evidence-based treatments; 2 ) research on the definition, occurrence, assessment, and treatment of fatigue through adequately funded prospective studies; and 3 ) providing optimal symptom control to all patients with cancer from diagnosis throughout the course of the illness. ${ }^{2}$ Although fatigue is the most common symptom of cancer, it is also the least understood. Cancer-related fatigue is reported by $60 \%$ to $99 \%$ of cancer patients and has been described as one of the most significant quality of life (QOL) issues in cancer care. $^{3,7-9}$ Patients with fatigue have significantly lower QOL, cognitive function, and physical performance. Similar to pain, numerous barriers to effective fatigue management have been documented. These include patient, professional, and system barriers.

\section{Patient Barriers}

Despite its prevalence and intensity, patients are reluctant to report fatigue and have little expectation that it can be relieved. ${ }^{2,8,10} \mathrm{~A}$ study involving 576 outpatients showed that patients who experience fatigue do not report it to their doctors because they feel it is inevitable $(43 \%)$, unimportant $(34 \%)$, or untreatable (27\%). ${ }^{8}$ Patients do not regard fatigue as a valid problem to complain about unless a health care provider specifically asks. ${ }^{7,9}$ Patients voluntarily complain of fatigue generally because it is either overwhelming or severely interfering with their lives. Patients report that they have difficulty communicating with their health care providers about their fatigue. ${ }^{11}$ When patients do report fatigue, they are often unsure about the process used for its diagnosis. They are eager to discover the cause of the problem but are distressed by the amount of time, money, and discomfort involved in the evaluation. ${ }^{12}$ The evaluation often leads to negative or "normal" results, suggesting to the patient that nothing can be done. Fatigue often causes patients to interrupt treatment schedules or stop treatment altogether, ${ }^{13,14}$ compromising the effectiveness of therapy and thereby potentially hindering the opportunity to treat the cancer. ${ }^{4,15}$ Neither patients nor their healthcare providers understand the mechanism of cancer-related fatigue or how to prevent, minimize, or resolve this pervasive and distressing symptom. These patient barriers create an imperative for professionals to take aggressive action in assessing and treating fatigue.

\section{Professional Barriers}

Fatigue is a symptom of cancer that is also poorly understood by professionals. ${ }^{4,16}$ This lack of knowledge stems from the complex nature of fatigue and the lack of agreement in the literature on the definition of fatigue and its causes, indicators, effects, or remedies. Health care providers place more emphasis on treating pain and nausea than fatigue, although fatigue has a very similar effect on QOL. Cancer-related fatigue is a symptom that is not routinely assessed in the clinical setting by health care providers ${ }^{10}$ and therefore is underreported, underdiagnosed, and undertreated. ${ }^{1}$ Even when patients report their fatigue, it may not be taken seriously by providers. ${ }^{11}$ Studies have reported that few patients ever receive treatment or advice from providers on how to manage their fatigue. ${ }^{3,8,16,17}$ Providers may erroneously assume that cancer-related fatigue is the same as healthy persons experience in everyday living, ${ }^{3,18}$ and therefore may not appreciate its significant negative effects. ${ }^{19,20}$ Providers may be unwilling to initiate discussion about fatigue with patients, particularly if they are unaware of available treatments ${ }^{2}$ or believe little can be done to manage it. A major barrier to fatigue management is the lack of knowledge about its underlying causes. ${ }^{2}$

\section{System Barriers}

Institutional and system barriers exist that are related to fatigue assessment and management. Documentation of fatigue assessment and management in the medical 
Borneman et al.

record is not a common requirement in most health care institutions and is not required by the Joint Commission on Accreditation of Healthcare Organizations. As a consequence, assessment and management of fatigue is often not a priority, and health care providers are not reminded that fatigue should be assessed and documented routinely. ${ }^{14-16}$ Using physical therapy to combat deconditioning is a good example of a potentially useful intervention affected by institutional/system barriers. For patients to receive physical therapy or occupational therapy, a physician's order is required to prevent indiscriminate use that could compromise care, such as physical therapy for a patient with metastatic bone disease. Unfortunately, because getting a physician's order can be burdensome, many patients are not referred. Health care reimbursement may also be a barrier, affecting the availability of medications, prescription practices, or referral patterns, such as for psychiatric or relational support, physical therapy, nutritional support, or erythropoietin therapy. ${ }^{19}$ Interventions are often implemented when fatigue is severe and patients are least able to participate in or benefit from them. More systematic approaches to treating fatigue are needed to overcome these barriers.

\section{Summary of Barriers to Fatigue Management}

The literature has documented that, similar to pain, significant patient, professional, and system barriers exist to the ideal management of fatigue based on clinical practice guidelines. Interventions are needed to improve patient and professional assessment and communication of fatigue. Professionals require increased knowledge of fatigue management, and systems are needed that provide timely, coordinated, and interdisciplinary approaches to fatigue management. After 2 decades of effort to advance symptom management, sources have documented that reducing these barriers requires more than providing guidelines or staff education. ${ }^{21,22}$ Education alone doesn't alter professional behavior, and professional change does not equate to patient/family or system change. For example, Allard et $a . .^{23}$ analyzed 33 studies of educational interventions for cancer pain and found that attitudes and knowledge about cancer pain improved, but unfortunately minimal change occurred in patients' pain. Ferris et al. ${ }^{24}$ recently summarized key steps to effective change by stating.

We can take the sum total of our evidence about education to improve cancer pain management in particular, and palliative care in general, and distill it to 3 take-home messages for those who want to pursue education: 1) design attitude and knowledge education that is practical and acknowledges the multiple other demands on the time of the learner; 2) design efforts to mentor and develop the desired skills using clinical settings; and 3) ensure that the systems are in place to facilitate and encourage the desired behaviors and outcomes.

\section{Research Framework}

Figure 1 schematically depicts the translational intervention model for fatigue. The Passport Model is a unique way to facilitate patient education in pain and fatigue management and to enhance the communication of symptoms to health care providers. At the top, barriers to effective management of fatigue are identified and categorized into patient, professional, and system barriers. ${ }^{1,2,25}$ Medical oncology patients are identified as eligible subjects, provided they have pain and/or fatigue ( $\geq 4$ on a $0-10$ screening scale) and meet other eligibility criteria. Demographic and disease variables that can affect fatigue and/or pain are identified. The 3 study phases are depicted, as are the intervening variables (disease progression and current treatments) that may affect outcomes. Phase I (baseline) documented usual care for pain and fatigue at accrual and 1 and 3 months past accrual. Phase II (high-intensity) involved intensive patient and professional education, with patient follow-up at 1 and 3 months after intervention. In phase III (low-intensity), investigators will continue to facilitate the intervention as it is implemented into existing systems and procedures within the cancer center. The barrier outcomes and how they are measured are displayed (patient, provider, and system).

\section{Methods}

\section{Design}

The first phase was designed to examine fatigue-related patient, professional, and system barriers that cause care to fall short of NCCN guidelines recommendations. This phase is followed by the intervention phases II and III. Several instruments used to measure fatigue at baseline were used to collect data.

\section{Sample}

Eligibility criteria included being 18 years of age or older; ability to read and understand English; and 
Implementing the NCCN Fatigue Guidelines

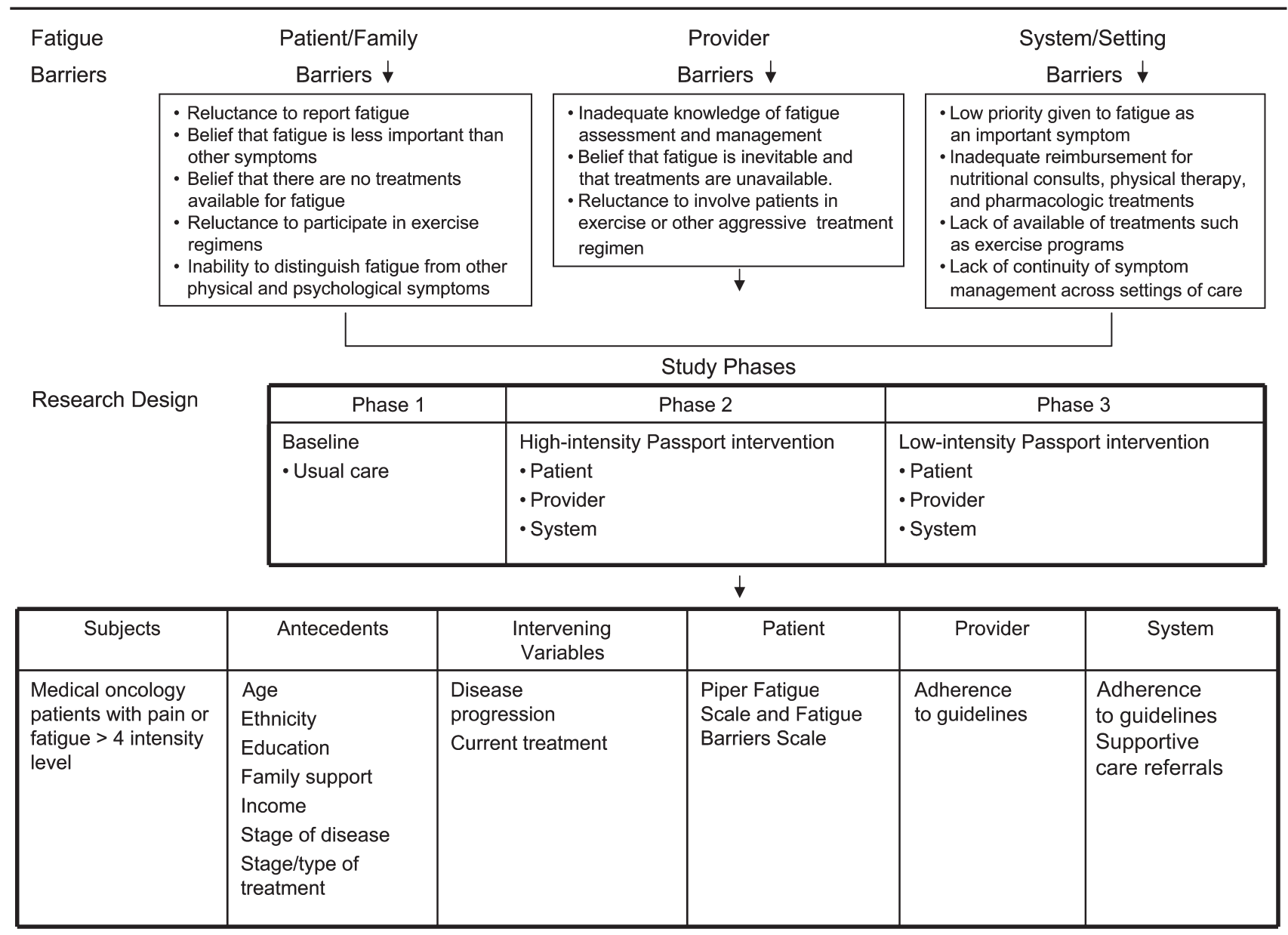

Figure 1 Translational Intervention Model for Fatigue: Passport to Comfort.

diagnosis of breast, lung, colon, or prostate cancer at least of 1 month before study entry (to avoid patients experiencing the distress of initial diagnosis, and to restrict patients to those with solid tumors with some degree of common treatment regimens and who are being seen and treated as outpatients). The study included subjects with a disease prognosis of 6 months or more to evaluate patients over time and avoid burdening individuals with later-stage disease who may experience rapid progression, and excluded those with fatigue ratings of $\leq 4$ on a 0 to 10 numeric screening scale to target those with moderate to severe symptom intensity as described in the NCCN guidelines. ${ }^{1}$

\section{Setting}

All data were collected at one medical oncology adult ambulatory care clinic at an NCI-designated comprehensive cancer center in Southern California.

\section{Instruments}

Demographic and Treatment Data Form: The investigator-developed demographic and treatment form was designed to capture key disease and treatment variables to describe the sample and analyze influencing variables. It consisted of 22 items measuring age, gender, education, and income level; type and stage of cancer; current and past cancer treatments; comorbidities and related medications; and Karnofsky Performance Status (KPS). ${ }^{26}$ Subjects completed the form at baseline, and items regarding stage and type of disease and treatment status were verified in the medical record by the research nurses.

Fatigue Intensity Scale: The Fatigue Intensity Scale (FIS) is an investigator-developed 11-item numeric rating scale that measures subjective fatigue on a 0 (no fatigue) to 10 (overwhelming fatigue) scale. This scale is similar to the screening tool recommended by the NCCN guidelines. It has face, content, and strong 
Borneman et al.

concurrent validity estimates when correlated with the standardized Piper Fatigue Scale (PFS) $(r=.83, P \leq$ $.001)^{27}$ and strong criterion validity $(r=.95$, $P \leq .0001)$ when tested with a comparable single-item fatigue-intensity scale in cancer patients undergoing chemotherapy, radiation therapy, hormonal therapy, and biologic therapies (Bookbinder M, Piper BF. Unpublished data from the MSKCC Fatigue Study Group). It was completed by patients as a screening tool to ensure that patients had moderate to severe levels of fatigue ( $\geq 4-10)$ to be eligible for the study. ${ }^{1}$ In this study, the FIS was modestly correlated with the PFS total score $(r=.37 ; P=.002)$, indicating that the FIS was a valid measure of fatigue in this study.

Piper Fatigue Scale: The PFS is a 22-item, self-report scale that measures 4 dimensions of subjective fatigue (behavioral/severity [6-items], sensory [5-items], cognitive/mood [6-items], and affective meaning [5-items]) confirmed through principal axes factor analysis with oblique rotation. ${ }^{28}$ Evidence for moderate to strong concurrent and discriminant validity estimates exist. Internal consistency (Cronbach's $\alpha$ ) reliabilities remain strong (0.83-0.97) for the PFS and its subscales across various cultural samples, languages, and diagnostic groups, and were 0.89 to 0.97 in this sample. Each item is measured on a 0 to 10 numeric rating scale, with opposing word anchors such as "not at all" to "a great deal." Items are summed and divided by the total number of scale items to keep scores on the 0 to 10 scaling. Higher scores indicate more fatigue. Mild (1-3), moderate (4-6), and severe (7-10) PFS total score levels have been validated with declines in physical functioning (Medical Outcome Study Short-Form 36 physical functioning subscale ). ${ }^{27}$ Five additional items, not included in the scale's scoring, are used to assess perceived causes, relief measures, additional fatigue descriptors, presence of other symptoms, and duration of fatigue. ${ }^{27,29}$ Patients completed the PFS at baseline.

Fatigue Barriers Scale: The Fatigue Barriers Scale is an investigator-developed 13-item scale designed to elicit patient beliefs and attitudes that might serve as barriers to effective fatigue assessment and management. It was developed based on an extensive literature review and clinical experience. ${ }^{1,2,25}$ Patients completed this scale at baseline. In this study, the reliability estimates for the subscales were beliefs/ attitudes $(\mathrm{r}=.30)$, good patient $(\mathrm{r}=.65)$, and fatalism $(\mathrm{r}=.54)$. For the total scale $(\mathrm{r}=.73)$, the reliability coefficient indicated good reliability for a new scale.
Fatigue Knowledge Scale: The Fatigue Knowledge Scale is an investigator-developed scale that contains 15 true and false statements about fatigue designed to assess a patient's knowledge about what fatigue is and how it can be assessed, measured, and treated. It was developed based on an extensive review of the literature and clinical experience ${ }^{1,25}$ and was designed to capture key potential patient-related knowledge barriers. Patients completed this scale at baseline.

Chart Audit Form: The audit form reflects the evidence-based recommendations contained within the NCCN Cancer-Related Fatigue Guidelines in Oncology for assessment and management and was used to capture the actual or usual care given by providers at baseline to document adherence to the guidelines preintervention. The form was pilot tested and revised before the start of phase 1 by the research nurses.

\section{Procedures}

The research nurses, who are both advance practice nurses, approached all patients who might be eligible for the study during regularly scheduled clinic visits. Once the study was explained and patients consented to participate, they were formally screened to ensure that all eligibility criteria were met. Patients completed all instruments at baseline (accrual).

\section{Data Analysis}

SPSS Sample Power and PASS power calculation software were used to estimate the sample size for this study based on the principal investigator's previous pain intervention studies. A sample of 50 patients was needed to provide $80 \%$ power to detect significance in the interaction effect of this statistical design, with a 2-tailed $\alpha$ of .05. Analyses included standard summary statistics of demographic and disease/treatment characteristics, and all scores at each period. Additional descriptive statistics were computed for all instrument subscale and total scores, including the KPS. Using data from the chart audit tool, frequencies of the NCCN recommended fatigue provider assessment and management behaviors were calculated. Multiple response frequencies were also calculated on support services ordered, comorbidities, and medications. Subscale and total scores were compared across selected demographic variables (race/ethnicity, education, religious preference, marital status, cancer diagnosis, and chemotherapy status) using multivariate analysis of variance (MANOVA) for subscales and ANOVA for total scores. Cronbach's $\alpha$ was used to determine the reliability estimates of selected scales. 
Implementing the NCCN Fatigue Guidelines

Subjects were given unique identification numbers, and a password-protected database was created. Subjects with missing scale or subscale scores were compared with those with nonmissing scores to ensure that no systematic bias caused the missing data (i.e., whether missing data were random or could be accounted for by other variables). For all data that were missing either completely or at random, the estimation-maximization method was used to replace missing values using the SPSS Replace Missing Values procedure.

\section{Results}

\section{Demographics}

The study accrued 100 subjects, of which 83 had complete evaluable data. At baseline, when patients were screened for eligibility using the FIS, 45 patients had fatigue only $(\geq 4) ; 24$ had both fatigue and pain $(\geq 4)$; and 14 had pain only $(\geq 4)$. For the purposes of this analysis, patients with fatigue only $(n=45)$ and both fatigue and pain $(n=24)$ were combined to form the study sample $(\mathrm{N}=69)$ used for all subsequent analyses.

Table 1 displays the demographic characteristics of the sample. The sample $(n=69)$ was predominantly Caucasian $(67 \%)$ women $(62 \%)$ who were married $(61 \%)$ and retired $(44 \%)$ with an annual income of more than $\$ 50,000$. The average subject was 60 years old, with moderate (rated 4-6) (55\%) or severe (rated $7-10)$ fatigue (45\%) and a KPS more than $80 \%$.

\section{Medical and Treatment Characteristics}

Table 2 summarizes the sample's medical and treatment characteristics. The most frequent malignancy was breast cancer (38\%), followed by lung, colorectal, and prostate cancer. The sample was somewhat evenly divided between being newly diagnosed (42\%) and having recurrent disease $(39.1 \%)$ with stage 3 or 4 disease on chemotherapy (78.3\%). The average hematocrit and hemoglobin levels were $35.04 \%$ and 11.83 $\mathrm{g} / \mathrm{dL}$, respectively. Most (72.5\%) were not receiving current supportive care services (i.e., social services, dietary, physical therapy, chaplaincy) nor had they tried complimentary or alternative therapies (87\%).

\section{Fatigue Findings}

Table 3 summarizes the PFS total and subscale scores for this sample at baseline. Mean scores ranged from 4.93 for the cognitive/mood subscale to 6.37 for the sensory subscale. The only statistically significant findings between demographic and medical variables and
Table 1 Demographic Characteristics $(\mathbb{N}=69)$

\begin{tabular}{|c|c|c|}
\hline Variables & Frequency(n) & Percentage (\%) \\
\hline \multicolumn{3}{|l|}{ Gender } \\
\hline - Male & 26 & 37.7 \\
\hline - Female & 43 & 62.3 \\
\hline \multicolumn{3}{|l|}{ Race/ethnicity } \\
\hline - Caucasian & 46 & 66.7 \\
\hline - African American & 7 & 10.1 \\
\hline - Asian & 4 & 5.8 \\
\hline - Hispanic/Latino & 10 & 14.5 \\
\hline - Other & 2 & 2.9 \\
\hline \multicolumn{3}{|l|}{ Martial status } \\
\hline - Married & 42 & 60.9 \\
\hline - Divorced & 14 & 20.3 \\
\hline - Widowed & 6 & 8.7 \\
\hline - Never married & 4 & 5.8 \\
\hline - Living with partner & 2 & 2.9 \\
\hline \multicolumn{3}{|c|}{ Education (highest achieved) } \\
\hline - High school & 28 & 40.6 \\
\hline - College & 26 & 37.7 \\
\hline $\begin{array}{l}\text { - Graduate/ } \\
\text { professional school }\end{array}$ & 14 & 20.3 \\
\hline \multicolumn{3}{|l|}{ Income } \\
\hline - $\leq \$ 10,000$ & 6 & 8.7 \\
\hline - $10,000-20,000$ & 4 & 5.8 \\
\hline - $20,001-30,000$ & 9 & 13.0 \\
\hline - $30,001-40,000$ & 6 & 8.7 \\
\hline - $40,001-50,000$ & 6 & 8.7 \\
\hline$\cdot \geq 50,000$ & 33 & 47.8 \\
\hline \multicolumn{3}{|l|}{ Religious preference } \\
\hline - Protestant & 29 & 42.0 \\
\hline - Catholic & 18 & 26.1 \\
\hline - Jewish & 4 & 5.8 \\
\hline - Buddhist & 1 & 1.4 \\
\hline - None & 5 & 7.2 \\
\hline - Other & 10 & 14.5 \\
\hline \multicolumn{3}{|l|}{ Current employment } \\
\hline - Full-time & 14 & 20.3 \\
\hline - Part-time & 5 & 7.3 \\
\hline - Retired & 30 & 43.5 \\
\hline $\begin{array}{l}\text { Retired because } \\
\text { of cancer }\end{array}$ & 4 & 5.8 \\
\hline - Homemaker & 5 & 7.2 \\
\hline $\begin{array}{l}\text { Unemployed on } \\
\text { disability because } \\
\text { of illness }\end{array}$ & 11 & 15.9 \\
\hline
\end{tabular}


Borneman et al.

\begin{tabular}{|c|c|c|c|c|}
\hline \multicolumn{5}{|l|}{ KPS } \\
\hline \multicolumn{2}{|l|}{ - 60} & 6 & \multicolumn{2}{|c|}{8.7} \\
\hline \multicolumn{2}{|l|}{ - 70} & 12 & \multicolumn{2}{|c|}{17.4} \\
\hline \multicolumn{2}{|l|}{ - 80} & 19 & \multicolumn{2}{|c|}{27.5} \\
\hline \multicolumn{2}{|l|}{ - 90} & 25 & \multicolumn{2}{|c|}{36.2} \\
\hline \multicolumn{2}{|l|}{ - 100} & 6 & \multicolumn{2}{|c|}{8.7} \\
\hline \multicolumn{5}{|c|}{ Fatigue Score (0-10) } \\
\hline \multicolumn{2}{|c|}{ - 4} & 8 & \multicolumn{2}{|c|}{11.6} \\
\hline \multicolumn{2}{|l|}{ - 5} & 21 & \multicolumn{2}{|c|}{30.4} \\
\hline \multicolumn{2}{|l|}{ - 6} & 9 & \multicolumn{2}{|c|}{13.0} \\
\hline \multicolumn{2}{|l|}{ - 7} & 17 & \multicolumn{2}{|c|}{24.6} \\
\hline \multicolumn{2}{|l|}{ - 8} & 11 & \multicolumn{2}{|c|}{15.9} \\
\hline \multicolumn{2}{|l|}{ - 10} & 3 & \multicolumn{2}{|c|}{4.3} \\
\hline Variable & Mean & SD & Minimun & Maximum \\
\hline - Age & 60.6 & 12.52 & 22 & 80 \\
\hline - KPS & 81.9 & 11.10 & 60 & 100 \\
\hline - Fatigue & 6.2 & 1.53 & 4 & 10 \\
\hline
\end{tabular}

Abbreviations: KPS, Karnofsky Performance Status; SD, standard deviation.

\begin{tabular}{|c|c|c|}
\hline Variables & Frequency $(n)$ & Percentage (\%) \\
\hline \multicolumn{3}{|l|}{ Type of malignancy } \\
\hline - Breast & 26 & 38 \\
\hline - Colon & 16 & 23 \\
\hline - Lung & 17 & 25 \\
\hline - Prostate & 10 & 14 \\
\hline \multicolumn{3}{|c|}{ Stage of disease (at diagnosis) } \\
\hline$\cdot \mathrm{IA}$ & 2 & 3 \\
\hline - \|A & 8 & 12 \\
\hline • IIB & 2 & 3 \\
\hline - IIIA & 10 & 15 \\
\hline - IIIB & 14 & 21 \\
\hline - IIIC & 5 & 8 \\
\hline$\bullet I V$ & 26 & 38 \\
\hline \multicolumn{3}{|l|}{ Current disease status } \\
\hline $\begin{array}{l}\text { - Newly diagnosed } \\
\text { under treatment }\end{array}$ & 29 & 42 \\
\hline $\begin{array}{l}\text { - Recurrent, under } \\
\text { treatment }\end{array}$ & 27 & 40 \\
\hline $\begin{array}{l}\text { - Completed } \\
\text { treatment } \\
\text { cancer-free }\end{array}$ & 1 & 2 \\
\hline - Other & 11 & 16 \\
\hline
\end{tabular}

\section{Table 2 Continued}

Current treatment status

- On chemotherapy

$\begin{array}{lll}\text { Yes } & 54 & 78 \\ \text { No } & 13 & 19\end{array}$

- On radiation therapy

$\begin{array}{lrr}\text { Yes } & 6 & 9\end{array}$

Previous treatment

- Surgery

$\begin{array}{lll}\text { Yes } & 48 & 70\end{array}$

$\begin{array}{lll}\text { No } & 20 & 29\end{array}$

- Chemotherapy

$\begin{array}{lll}\text { Yes } & 62 & 90\end{array}$

$\begin{array}{lll}\text { No } & 6 & 9\end{array}$

- Radiation therapy

$\begin{array}{lll}\text { Yes } & 31 & 45\end{array}$

$\begin{array}{lll}\text { No } & 37 & 54\end{array}$

Comorbidities

- Heart disease $12 \quad 12$

- Hypertension 2423

- Anxiety/depression $10 \quad 1$

- Diabetes 8

- Arthritis 6

- Obesity 6

- Other $30 \quad 30$

Variable(s) Mean SD Minimum Maximum

- Number of years since initial cancer $\begin{array}{lllll}\text { diagnosed } & 3.22 & 3.32 & 1.0 & 15.0\end{array}$

- Number of years since starting $\begin{array}{lllll}\text { chemotherapy } & 1.28 & 2.93 & -0.87 & 14.0\end{array}$

- Number of years since starting $\begin{array}{lllll}\text { radiation } & 2.09 & 2.63 & -0.87 & 10.28\end{array}$

$\begin{array}{lllll}\text { - Hemoglobin level } & 11.83 & 1.56 & 8.3 & 15.8\end{array}$

$\begin{array}{lllll}\text { - Hematocrit level } \quad 35.04 & 4.17 & 25.7 & 44.3\end{array}$

Abbreviations: SD, standard deviation.

PFS scores were income level, number of medications being taken, and self-reported KPS. Patients with lower incomes (i.e., $<\$ 50,000$ vs. $>\$ 50,000$ ) had significantly more severe fatigue as measured with the PFS behavioral severity subscale $(P=0.04)$. Patients who were taking more medications had significantly higher affective $(P=.003)$, behavioral $(P=.046)$, and 
Implementing the NCCN Fatigue Guidelines

\begin{tabular}{|lll|}
\hline $\begin{array}{l}\text { Table } 3 \\
\text { Piper Fatigue Scale Total and Subscale } \\
\text { Scores (Range, } 0-10)(\mathrm{N}=68) *\end{array}$ \\
\hline Scale/Subscale & Mean & SD \\
\hline Sensory & 6.37 & 2.20 \\
Affective meaning & 5.91 & 2.34 \\
Cognitive mood & 4.93 & 2.05 \\
Behavioral severity & 5.98 & 2.61 \\
PFS total & 5.76 & 2.05 \\
\hline
\end{tabular}

*Note: One subject had missing items on this scale, so these scores were dropped from the analysis.

Abbreviations: PFS, Piper Fatigue Scale; SD, standard deviation.

total PFS scores $(P=.03)$, and patients who had higher KPS scores had less fatigue as measured with the PFS total $(\mathrm{r}=-.35 ; P=.003)$ and FIS scores $(\mathrm{r}=-.41$; $P=.001)$. Patients with more comorbidities had significantly higher behavioral fatigue $(r=.25 ; P=.038)$. Other demographic/medical characteristics (i.e., age, gender, educational level, ethnicity, religious affiliation, anemia, or current computed tomography) were not related to fatigue.

\section{Patient Barriers}

Fatigue Barriers Scale: The most frequent patientrelated barrier at baseline included believing that the doctor would ask about fatigue if it was important (54\%). In terms of demographic/medical characteristics, an increased number of fatigue barriers was associated with less education $(P=.01)$ and playing the "good" patient role $(P=.001)$. Having less income $(<\$ 50,000)$ was associated with having more fatalistic beliefs about fatigue $(P=.006)$, as was not being married or partnered $(P=.008)$. Patients with colon cancer had more negative belief barriers to fatigue than those with lung cancer $(P=.018)$. Patients who were 4 or more years out from their initial diagnosis were also more likely to have more negative beliefs $(P=.008)$ and an increased number of barriers $(P=$ $.030)$ in general than patients who were more recently diagnosed. Patients who had between 3 and 7 comorbidities scored significantly higher on the "good patient" and fatalism barriers than patients who had 1 or 2 comorbidities. No significant relationships were documented between fatigue barriers and other demographic/medical characteristics (e.g., gender, ethnicity, age, religion, chemotherapy status, number of medications).

When patients were asked what they believed most directly contributed to or caused their fatigue, the most common responses were chemotherapy, treatments or side effects of treatments, and medications. When asked what best relieved their fatigue, the most frequent responses included rest, sleep, and trying to perform various activities, such as walking. Distraction and socializing were also common relief strategies. When asked to better describe their fatigue, many stated that they were not able to do the kinds of activities that they enjoyed doing. One patient even stated that fatigue was "destructive" to the life of a normally active person. Common symptoms also included pain, nausea, difficulty sleeping, and gastrointestinal problems (e.g., diarrhea, constipation, irritable bowels). When asked if any aspect of their fatigue hadn't been captured, one patient stated that the fatigue "just suddenly hits me like running into a brick wall," and another stated that "it's keeping me from accomplishing the things I want to do before I die." Not being able to do specific activities with family and friends was a common response given for how fatigue negatively affected their quality of life.

Fatigue Knowledge Scale: Overall, patient knowledge was accurate (i.e., 84\%-95.1\% correct); the worst scores related to the exercise items: "Exercise reduces energy, which leads to more effort when you do your usual activities" (72\% correct), and "If you are really tired, you should stay in bed all day" (78\% correct). Knowledge about fatigue was positively associated with a higher income levels $(P=.004)$, but no other demographic or medical characteristic (e.g., age, education, religion, marital status, cancer diagnosis, time since diagnosis, chemotherapy treatment).

\section{Provider and System Barriers}

Overall, the chart audit data conducted at baseline reflected a lack guideline adherence, fatigue documentation, and supportive care referrals (Table 4 ). The highest adherence was documented for anemia assessments $(28.99 \%)$, followed by fatigue screening at the visit (23.14\%), nutritional assessment (20.29\%), review of systems (18.84\%), and comorbidity assessment $(18.84 \%)$.

\section{Discussion}

Findings indicated that several patient-, provider-, and system-related barriers exist to effective fatigue management at baseline. Additionally, evidence shows 
Borneman et al.

\section{Table 4 Chart Audit Findings at Baseline $(\mathrm{N}=69)$ \\ Guideline Adherence Demonstrated}

Screened for fatigue at visit

Screening for fatigue is consistent

Fatigue rating documented

Education provided if patient had none to mild fatigue

Focused history conducted for moderate to severe fatigue

Review of systems performed for moderate to severe fatigue

In-depth assessment conducted for

moderate to severe fatigue

Pain assessment preformed

Emotional distress assessment performed

Sleep problem assessed

Anemia assessed

Nutrition assessed

Activity level assessed

Comorbidity assessed

Education provided regarding:

- Fatigue patterns

- Daily self monitoring

- Energy conservation

- Activity enhancement

- Sleep hygiene

- Restorative therapy

- Stress management

- Relaxation methods

Referrals made to:

- OT/PT

- Nutritionist

- Psychosocial support

- Support groups

Pharmacologic intervention:

- Psychotropic

$\%$ Yes

23.19

8.70

2.90

0

1.45

18.84

1.45

15.94

5.80

11.59

28.99

20.29

17.39

18.84

Follow-up evaluations between visits

Abbreviations: OT, occupational therapist; PT, physical therapist.

that the NCCN Cancer-Related Fatigue Clinical Practice Guidelines in Oncology were not being translated into practice. The authors suspect that these findings are not unique to this setting or its healthcare providers.
The most frequent patient-related barrier to effective fatigue management was the patient's belief about relying on the doctor to introduce the subject of fatigue if it was important. Passik et al..$^{30}$ reported a similar finding, with $66 \%$ of patients stating that they had never even spoken to their doctor about fatigue. In addition, more barriers were associated with having less education, lower income levels, not being married/partnered, more comorbidities, and being 4 or more years out from their initial diagnosis. This suggests that tailored educational efforts to improve fatigue communication between patients and providers to high-risk groups may be necessary to break down the communication barriers that may exist in practice settings.

\section{Limitations}

This study was implemented at one National Comprehensive Cancer Center outpatient setting located in Southern California where patients are commonly referred for treatment and second opinions, usually late in the course of their treatment and disease process. As a consequence, the findings may not be generalizable to other geographic areas or inpatient settings, or to other populations, such as newly diagnosed patients with early stage disease, survivors without evidence of disease, or patients with other types of malignancies. The physicians who participated in this study were all medical oncologists who saw the patients during routine follow-up appointments.

\section{Research Implications}

This is the first study that attempts to document barriers to the translation of and adherence to the NCCN Cancer-Related Fatigue Guidelines in practice. Continued research is warranted to develop educational measures for both patients and providers that are effective in decreasing the barriers that exist to effective communication and treatment of fatigue. Additional research is needed to determine if these educational efforts make a difference in fatigue assessment, documentation, and patient outcomes. In phase 2 of this 3-phased trial, a "high-intensity educational intervention" will be tested for patients and providers to see what effect these strategies have on ameliorating the many barriers that exist in practice settings to effective fatigue management. 
Implementing the NCCN Fatigue Guidelines

\section{Practice Implications}

This study's findings reflect the need to implement educational strategies for both patients and providers to improve fatigue knowledge, management strategies, and referral patterns for better management of fatigue. The hope is that the major outcome of this 3-phased trial will be a model for educating patients and providers that can be easily translated into other practice settings.

\section{References}

1. Mock V, Abernathy AM, Atkinson A, et al. The NCCN CancerRelated Fatigue Clinical Practice Guidelines, version 3.2007. Available at: www.nccn.org/professionals/physician_gls/PDF/fatigue.pdf. Accessed December 5, 2006.

2. NIH State-of-the-Science Statement on symptom management in cancer: pain, depression, and fatigue. NIH Consens State Sci Statements 2002;19:1-29.

3. Vogelzang NJ, Breitbart W, Cella D, et al. Patient, caregiver, and oncologist perceptions of cancer-related fatigue: results of a tripart assessment survey. The Fatigue Coalition. Semin Hematol 1997; 34(Suppl 2):4-12.

4. Winningham ML, Nail LM, Burke MB, et al. Fatigue and the cancer experience: the state of the knowledge. Oncol Nurs Forum 1994;21:23-36

5. Klausner RD. The Nation's Investment in Cancer Research: A Budget Proposal for Fiscal Year 1999. Bethesda, MD: National Cancer Institute; 1997.

6. Portenoy RK, Miaskowski C. Assessment and management of cancer-related fatigue. In: Berger AM, Portenoy RK, Weissman DE, eds. Principles and Practice of Supportive Oncology. Philadelphia, PA: Lippincott-Raven; 1998:109-118.

7. Schwartz AL, Nail LM, Chen S, et al. Fatigue patterns observed in patients receiving chemotherapy and radiotherapy. Cancer Invest 2000;18:11-19.

8. Stone P, Richardson A, Ream E, et al. Cancer-related fatigue: inevitable, unimportant and untreatable? Results of a multi-centre patient survey. Cancer Fatigue Forum. Ann Oncol 2000;11:971-975.

9. Cella D, Peterman A, Passik S, et al. Progress toward guidelines for the management of fatigue. Oncology (Williston Park) 1998;12:369-377.

10. Nail L. Fatigue in patients with cancer. Oncol Nurs Forum 2002;29:537-546.

11. Grant M, Golant M, Rivera L, et al. Developing a community program on cancer pain and fatigue. Cancer Pract 2000;8:187-194.

12. Dean G, Anderson P. Fatigue. In: Ferrell B, Coyle N, eds. Textbook of Palliative Nursing. Oxford: Oxford University Press; 2001:91-100.

13. Ancoli-Israel S, Moore PJ, Jones V. The relationship between fatigue and sleep in cancer patients: a review. Eur J Cancer Care (Engl) 2001;10:245-255.
14. de Jong N, Courtens AM, Abu-Saad HH, Schouten HC. Fatigue in patients with breast cancer receiving adjuvant chemotherapy: a review of the literature. Cancer Nurs 2002;25:283-297; quiz 298-299.

15. Payne JK. The trajectory of fatigue in adult patients with breast and ovarian cancer receiving chemotherapy. Oncol Nurs Forum 2002; 29:1334-1340.

16. Visser MR, Smets EM. Fatigue, depression and quality of life in cancer patients: how are they related? Support Care Cancer 1998; 6:101-108.

17. Passecreta J. Measuring depression. In: Frank-Stromborg M, Olsen SJ, eds. Instruments for Clinical Health-Care Research. 3rd ed. Boston, MA: Jones \& Bartlett; 2004:376-400.

18. Glaus A, Crow R, Hammond S. A qualitative study to explore the concept of fatigue/tiredness in cancer patients and in healthy individuals. Support Care Cancer 1996;4:82-96.

19. Nail LM. Long-term persistence of symptoms. Semin Oncol Nurs 2001;17:249-254.

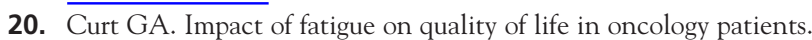
Semin Hematol 2000;37:14-17.

21. Slotnick HB. How doctors learn: physicians' self-directed learning episodes. Acad Med 1999;74:1106-1117.

22. Davis D, O'Brien MA, Freemantle N, et al. Impact of formal continuing medical education: do conferences, workshops, rounds, and other traditional continuing education activities change physician behavior or health care outcomes? JAMA 1999;282:867-874.

23. Allard P, Maunsell E, Labbe J, Dorval M. Educational interventions to improve cancer pain control: a systematic review. J Palliat Med 2001;4:191-203.

24. Ferris FD, von Gunten CF, Emanuel LL. Knowledge: insufficient for change. J Palliat Med 2001;4:145-147.

25. Passik SD. Impediments and solutions to improving the management of cancer-related fatigue. J Natl Cancer Inst Monogr 2004;32:136.

26. Karnofsky D, Burchenal J. The clinical evaluation of chemotherapeutic agents in cancer. In: Macleod CM, ed. Evaluation of Chemotherapeutic Agents. New York, NY: Columbia University Press; 1948:199-205.

27. Piper BF, Dodd MJ, Ream E, et al. Improving the clinical measurement of cancer-related fatigue. In: Better Health Through Nursing Research: International State of the Science. Washington, DC: American Nurses Association; 1999:99.

28. Piper BF, Dibble SL, Dodd MJ, et al. The revised piper fatigue scale: psychometric evaluation in women with breast cancer. Oncol Nurs Forum 1998;25:677-684.

29. Piper BF. Fatigue. In: Frank-Stromborg M, Olsen SJ, eds. Instruments for Clinical Health-Care Research. 3rd ed. Boston, MA: Jones \& Bartlett; 2004:538-569.

30. Passik SD, Kirsh KL, Donaghy K, et al. Patient-related barriers to fatigue communication: initial validation of the fatigue management barriers questionnaire. J Pain Symptom Manage 2002;24:481-493. 